\title{
Effect of acute and delayed remote ischemic preconditioning on systemic inflammatory response in a septicemia model
}

\author{
Choon Hak Lim, Seol Ju Park, Min Kyung Park, Yun Hee Kim \\ Department of Anesthesiology and Pain Medicine, Korea University Medical Center, Seoul, Korea
}

\begin{abstract}
Remote ischemic preconditioning (RIPC) has a protective effect on ischemic-reperfusion (I/R) injury and septic inflammatory injury. The protective effect of preconditioning has been reported to have a biphasic pattern in $\mathbf{~} / \mathbf{R}$ injury; acute protective effects wane after a few hours, but a delayed second window of protection occurs after $24 \mathrm{~h}$. This study assessed whether delayed RIPC can improve the survival rate and the length of effectiveness of the delayed period in a mouse model of lipopolysaccharide (LPS) induced sepsis. RIPC was performed with three cycles of $10 \mathrm{~min}$ of ischemia and $10 \mathrm{~min}$ of reperfusion of the right hind limbs using a tourniquet $0,1,4,12$, and 24 hours prior to intraperitoneal (i.p.) injection of $20 \mathrm{mg} / \mathrm{kg}$ LPS. Other mice received just i.p. LPS. The control group received i.p. normal saline. The 5-day survival rate, serum cytokine levels and liver neutrophil infiltration were measured. Survival was significantly increased in mice who received RIPC compared to the LPS group (80-100\% vs $30 \%, P<0.05)$. Levels of interleukin-6 and -12 were significantly decreased in with all times of RIPC compared to the LPS group (all $P<0.05$ ). Neutrophil infiltration was substantially attenuated in all RIPC treated groups prior to LPS injection. The results demonstrate that acute, subacute and delayed RIPC attenuates the inflammatory response and improves survival of mice with LPS-induced septicemia.
\end{abstract}

Remote ischemic preconditioning (RIPC) induced by transient brief episodes of ischemia and reperfusion of tissue or organ is a powerful protective mechanism of remote tissues or organs against a subsequent and more severe ischemia-reperfusion (I/R) insult. Transmission of the RIPC signal to the target organ is probably multi-factorial, requiring a combination of humoral, neuronal, and systemic modification of circulating immune cells and activation of hypoxia inducible genes. The protective effect of preconditioning has been reported to display a biphasic pattern in which the acute protective effects wane after a few hours, with a delayed second window of protection occurring after $24 \mathrm{~h}$. The acute effects rely on the activation of existing signaling molecules, whereas the delayed effects are achieved by increased expression of protective proteins. RIPC and remote ischemic post-conditioning performed immediately before injection of lipopolysaccharide (LPS) reduces pro-inflammatory cytokines and active neutrophils, and improves survival outcomes in a model of sepsis. Sepsis is responsible for high rates of morbidity and mortality among intensive care unit (ICU) patients. It is difficult to screen for sepsis and take measures to attenuate the septic response in the susceptible patients. So, RIPC could be a clinically feasible and beneficial strategy for sepsis. However, it is not clear when preconditioning should be done and whether RIPC exhibits a biphasic pattern in clinical cases of sepsis. We hypothesized that acute or delayed RIPC both have an antiinflammatory response in a mouse model of sepsis. The study assessed the protective capacity and survival improvement of delayed RIPC and how long delayed period could be effective in a LPS-induced sepsis model.

Six-week-old male BALB/c mice (Hanlim Co. Ltd., Hwasung, South Korea) were used. The LPS-induced sepsis model was created by injecting $20 \mathrm{mg} / \mathrm{kg}$ LPS intraperitoneally (i.p.). The commercial preparation of LPS from Escherichia coli O127: B8 (Sigma-Aldrich, St. Louis, MO, USA) was dissolved in $0.5 \mathrm{~mL}$ physiological saline. The mice in the LPS and RIPC/LPS groups (see below) were each injected i.p. with $0.5 \mathrm{~mL}$ of the LPS solution. Mice in the control group received $0.5 \mathrm{~mL}$ i.p. saline. RIPC was induced with three cycles involving $10 \mathrm{~min}$ of ischemia and $10 \mathrm{~min}$ of reperfusion of the right hind limbs using a tourniquet. Ischemia was judged to have occurred if the affected leg looked pale. The mice were randomly divided into seven experimental groups. The control (saline) group is described above. The LPS group received $20 \mathrm{mg} / \mathrm{kg}$ LPS via i.p. injection. The remaining five groups received RIPC $0,1,4,12$, or $24 \mathrm{~h}$ prior to i.p. injection of $20 \mathrm{mg} / \mathrm{kg}$ LPS (RIPC 0H/LPS, RIPC 1H/LPS, RIPC 4H/LPS, RIPC 12H/LPS, and RIPC 24H/LPS, respectively) (Fig. 1).

Of the 10 mice in each group, 10, 3, 9, 10,10, 8, and 10 survived in the Saline, LPS, RIPC 0H/LPS, RIPC 1H/LPS, RIPC 4H/LPS, RIPC 12H/LPS, and RIPC 24H/LPS groups, respectively. The 5-day survival rate of the Saline and all RIPC/LPS groups was significantly greater than the LPS group ( $\mathrm{P}=0.001,0.005,0.001,0.001,0.02$, and 0.001 , respectively). There was no significant difference among the Saline and RIPC/LPS groups

Copyright @ 2017 Choon Hak, Lim, et al (yourejoice@korea.ac.kr) respectively).
IL-6 levels in RIPC 1H/LPS, RIPC 4H/LPS and RICP 12H/LPS groups were significantly lower than the LPS group $(\mathrm{P}=0.002,0.009$ and 0.0009 , respectively). IL-12 levels in all RIPC/LPS groups were significantly lower than the LPS group $(\mathrm{P}=0.02,0.009$ and 0.009 ,

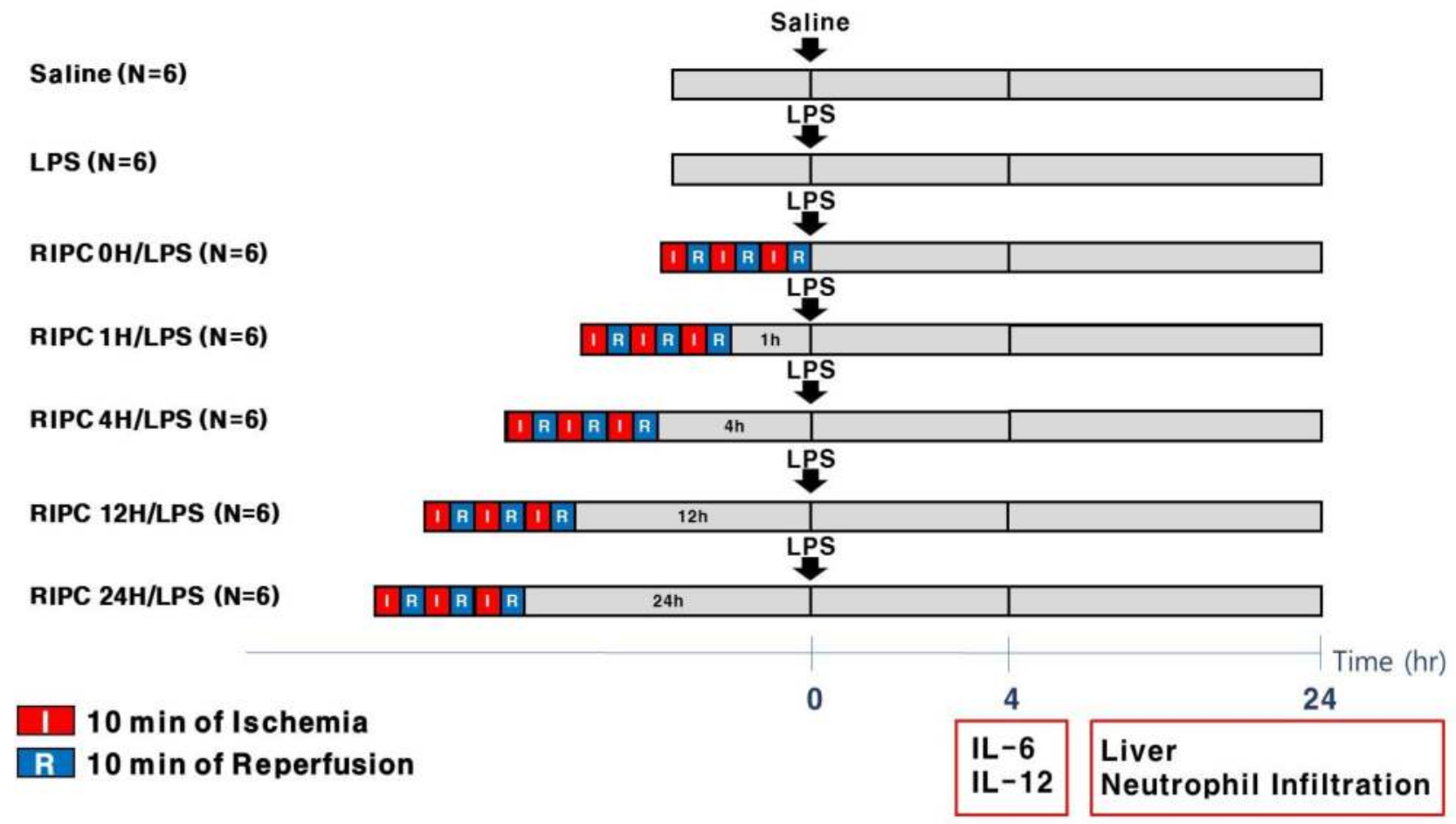

Figure 1. Experimental Group.

Quantification of intrahepatic sinusoidal neutrophils by counting the brown-stained PMNs in the all RIPC group revealed a substantially decreased neutrophil count compared to the LPS group but not significantly, lower than the LPS group $(\mathrm{P}=0.84)$.

The results suggest the potential benefit of early, subacute, and delayed RIPC would be a new early treatment during sepsis. Intermittent pneumatic compression is routinely applied to ICU patients to prevent deep vein thrombosis and might be used as an alternative to RIPC by virtue of its low-cost and non-invasive nature. However, there are discrepancies in immunity between human and mice, so findings with mice, including the present findings, should be carefully interpreted in term of the possible clinical benefit to humans. In addition, it is not clear why RIPC did not have biphasic effects in the present LPSinduced sepsis model, unlike the I/R injury model, and which mechanism is different. Further study is needed.

In conclusion, the present study demonstrates that acute, subacute, and delayed RIPC attenuates LPS-induced sepsis in a mouse model. The benefits include reduced pro-inflammatory cytokines IL-6 and IL-12, reduced neutrophil infiltration in the liver, and improved survival. 